\title{
Study on the Preparation of Coal Water Slurry and Its Additives
}

\author{
Zhang Yanmin, Guo Guozhe, Li Yi, Wang Lipeng, Zhang Lala \\ College of Chemistry and Chemical Engineering, Longdong University, Qingyang, 745000
}

Keywords: coal water slurry; sodium lignosulfonate; sodium dodecyl sulfate

\begin{abstract}
Coal water slurry (CWS) is a new type of fuel with good rheology and stability. In this paper, the effects of two dispersants on the viscosity and water separation rate of coal water slurry were studied. The results show that when the CWS concentration is $67 \%$, the viscosity of coal slurry added with sodium dodecyl sulfate is $986 \mathrm{mpa} \cdot \mathrm{s}$, and the viscosity of coal slurry added with sodium lignosulfonate is $1001 \mathrm{mpa} \cdot \mathrm{s}$. Adding sodium lignosulfonate coal water slurry has better hydrophilicity and higher stability.
\end{abstract}

\section{Introduction}

China is a country with rich coal resources and poor oil resources. Since China became a net exporter in 1993, its dependence on imports has been increasing. Now it has become the second largest oil consumer in the world after the US. The annual growth rate of oil imports ranks first in the world. Coal resources account for more than $65 \%$ of China's energy consumption. However, the traditional coal resource utilization has many disadvantages, such as lots of ash, heavy pollution, difficult transportation and low heating rate. Coal water slurry (CWS), as an important clean energy, can effectively reduce environmental pollution ${ }^{[1]}$. It was a coal based liquid fuel that rose in 1970s. Coal water slurry used to replace oil and replace coal with good energy-saving and environmental benefits. Coal water slurry (CWS) technology is a major reform for the deep processing and rational utilization of coal resources ${ }^{[2]}$.

Coal water slurry is a new type of fuel that is obtained by physical method. It not only keeps the original physical characteristics of coal, but also has the same liquidity and stability as oil. It is called liquid coal product. In recent years, because of the abundant coal resources and low price, the processing method of coal water slurry is simple. Compared with coal gasification and liquefaction, it has less investment and good stability. The cost of making coal water slurry is low, and it has been paid much attention by all countries in the world as oil generation fuel. The coal water slurry with industrial application value should have high concentration, low viscosity, good fluidity and stability. Therefore, a proper amount of chemical additives should be added to the mixture of coal and water ${ }^{[3]}$. The dispersants used for pulping are all surface active agents. One end is a nonpolar pro-oil groups made up of hydrocarbons and the other is a hydrophilic polar base ${ }^{[4-6]}$.

In China, the technology of coal water slurry commonly exists many problems such as the matching of additives and coal. A kind of additive for coal are only specific to dispersion viscosity, while the other coal poor effect, seriously restricting the promotion of additives, which greatly reduces the economic benefits of new products, so a wide area of additive is the current and future research direction.

According to different functions, coal water slurry additive can be divided into dispersant, stabilizer (such as gelatin) and some auxiliary agents (such as surface active agent, defoaming agent, $\mathrm{PH}$ regulator, mildewcide etc.). Dispersant is the most important. The main function of the dispersant is to change the surface properties of the coal particles and promote the dispersion of the particles in the water, so that the slurry has a good rheological property. The mechanism of its action can be explained in three aspects: wetting dispersant, electrostatic repulsion and steric hindrance.

According to the dissolution of water, the dispersant of water coal slurry can be divided into anionic, cationic, nonionic and amphoteric types. At present, the main dispersants developed and screened at home and abroad are anionic and nonionic. There are mainly naphthalene system, humic 
acid system, lignin system, polyolefin system, acrylic acid system, and the related compound products are developed for different kinds of coal.

Other auxiliary chemical reagents used for coal water slurry include: defoaming agent, surface active agent, $\mathrm{PH}$ regulating agent, mildewcide and accelerator etc. They are not necessary during the production of coal water slurry. Their use has a great relationship with the additives of coal and pulping. For example, when the pulping coal is used for flotation clean coal, there will be a lot of bubbles when stirred by more foaming agents on the surface. If the non ionic additives are used for pulping, they are also good foaming agents. There are too many bubbles in coal water slurry, which will greatly affect its fluidity. At this point, the defoaming agent needs to be added to eliminate its influence. For some coal which is difficult to make pulp, some surface active agents should be added to the pulping process to change the surface characteristics of the coal particles so as to enhance their pulping ${ }^{[7-8]}$.

The stability of coal water slurry (CWS) refers to the uniformity of the coal slurry during storage and transportation. Because coal water slurry is a solid and liquid coarse dispersion system, coal particles are easily coalescing spontaneously, under the action of gravity or other external forces, it is easy to precipitate. A stabilizer is added to the coal slurry in order to prevent precipitation or to form an non recoverable hard precipitation. A thixotropic body is formed by the coarse grained suspension of coal water slurry. That is, when coal water slurry is placed in a static way, it is structured and has high shear stress. Once external force is applied, the viscosity of coal water slurry can be rapidly reduced, and it has good fluidity. When the CWS is placed again, it will restore its original structure state. Stabilizers are those chemical reagents which make the coal particles dispersed in coal water slurry and other coal particles and water around them form a weaker, but also a certain intensity of three-dimensional structure. The addition of stabilizers can cross - link the dispersed solid particles and form a space structure, thus effectively preventing the particles from settling and preventing the separation of solid and liquid. At the present stage, the main stabilizers of coal water slurry are two kinds of inorganic electrolyte and polymer compound. Such as all kinds of soluble salts, polymer surfactants, cellulose, polyacrylate, and some colloid particles (such as organic bentonite) etc ${ }^{[9]}$.

\section{Experimental Part}

Sodium lignosulfonate as dispersant has advantages of abundant raw materials and low cost. Therefore, sodium lignosulfonate and sodium dodecyl sulfate are selected as additives for coal water slurry. First, the viscosity was measured under the concentration of different coal water slurry, the coal water ratio is determined most suitable for this experiment. On this basis, the influence of the dosage of different dispersing agent on the viscosity and water evolution of coal water slurry is analyzed, and the stability analysis is carried out ${ }^{[10]}$. According to the data, the conclusion of the experiment is finally obtained.

According to the experimental ratio, the distilled water was added into the corundum bottle and added dispersant. The mixture was stirred for $5 \sim 10 \mathrm{~min}$ in the agitator, and the prepared coal sample was added to the agitated solution, and then the mixture was stirred for $10 \sim 15 \mathrm{~min}$ until the solution was pulping.

Table 1 Experimental instruments

\begin{tabular}{lll}
\hline Instrument name & Model & \multicolumn{1}{c}{ Manufacturer } \\
\hline Electronic balance & GH224S & Beijing Sai duo li si Instrument System Co. Ltd. \\
Jaw crusher & EP-1 & He bi Feng tai Instrument and Instrument Co., Ltd. \\
Rotary viscometer & NDJ-8S & $\begin{array}{l}\text { Shanghai Ping xuan Scientific Instruments Co., } \\
\text { Ltd. } \\
\text { Changzhou Guo hua Electrical Appliance Co., Ltd. }\end{array}$ \\
Electric Blender & JJ-1 & \\
\hline
\end{tabular}


Table 2 Experimental reagents

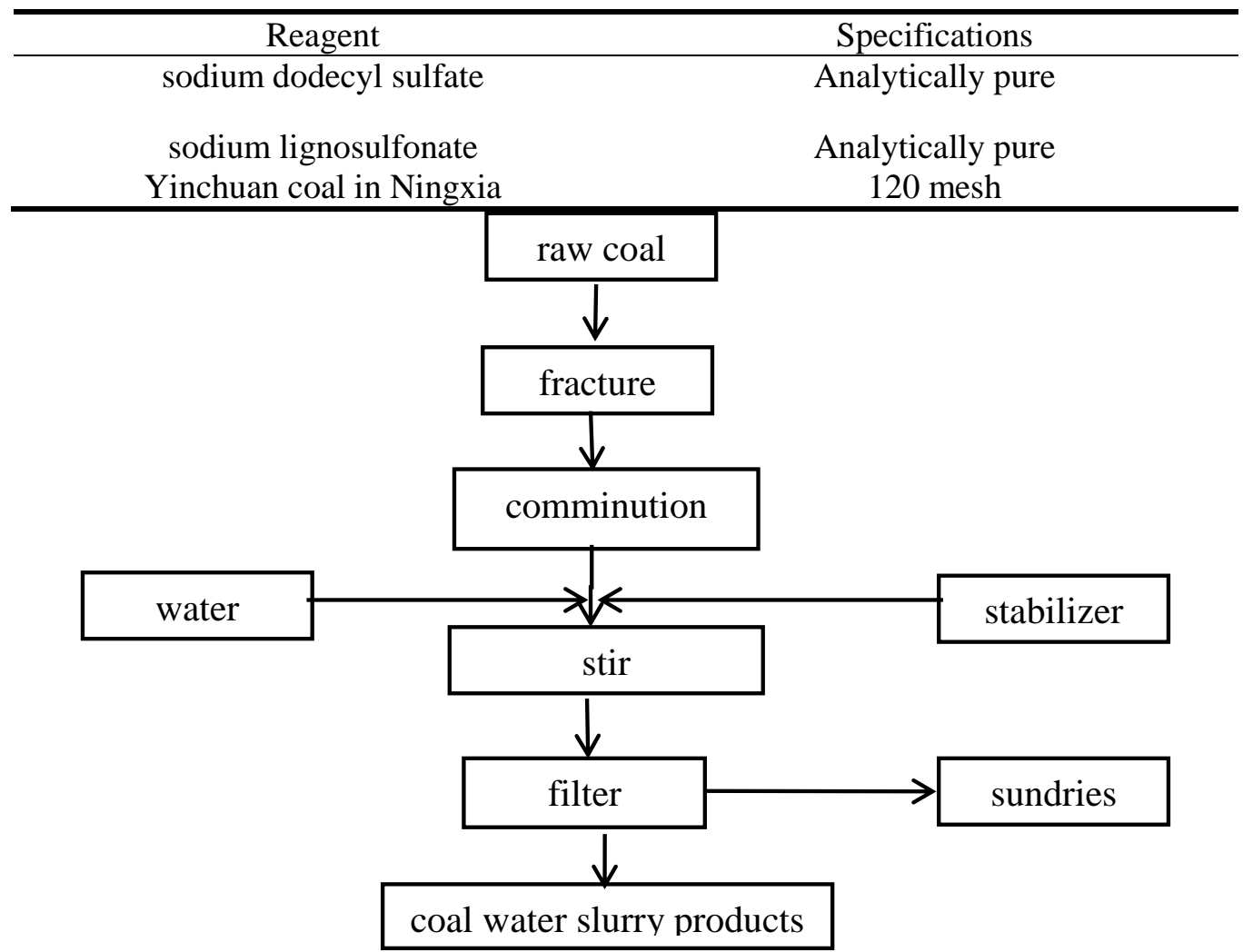

Figure 1 Process flow chart of coal water slurry preparation

In this experiment, the viscosity of coal water slurry is measured by a NDJ-8S rotary viscometer with a coaxial cylinder measuring system.

Operation steps:

(1) The measured liquid is placed in a beaker or cylindrical container with a diameter of no less than $70 \mathrm{~mm}$ and no less than $125 \mathrm{~mm}$.

(2) The temperature of the measured liquid is controlled accurately.

(3) Refer to the range table, select the suitable rotor to spin into the rotor connection head.

(4) Adjust the lifting knob slowly and adjust the height of the rotor in the measured liquid until the middle of the groove of the rotor is flat with the liquid level.

(5) Adjust the level of the instrument, check whether the bubble in the instrument is in the middle, and ensure that the instrument is in a horizontal working state.

(6) Select the rotor by the rotor key, press the definite key.

(7) After the value is stable, 10 sets of values are read, and the average value is calculated and recorded as the final viscosity.

(8) Test the experiment, turn off the power, take the rotor and clean it, put it back.

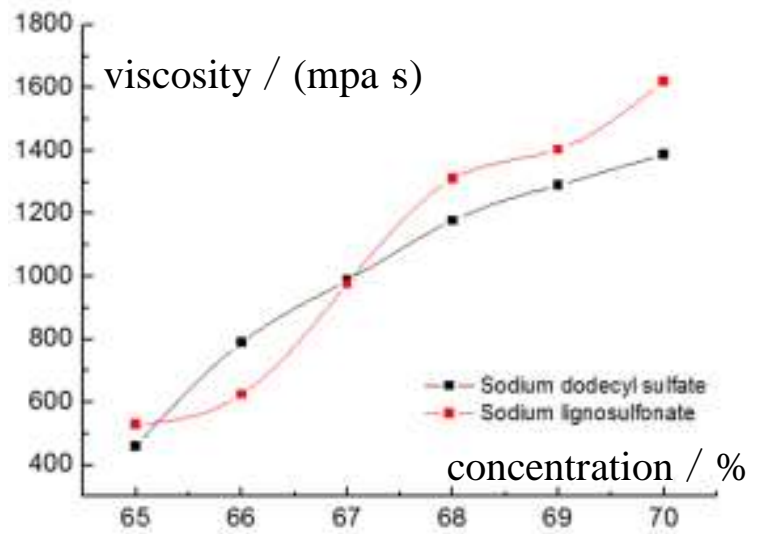

Figure 2 The influence of the concentration of coal water slurry on apparent viscosity 
The content of the additive is $1 \%$. Coal water slurry with a concentration of $65 \%, 66 \%, 67 \%$, $68 \%, 69 \%$ and $70 \%$ was placed at room temperature for 12 hours. Then the viscosity was measured. The results of the experiment are shown in Figure 2.

From Fig. 2, it is known that the viscosity of two kinds of coal water slurry increases with the increase of CWS concentration. When the concentration of CWS is after $67 \%$, the viscosity of CWS produced by sodium lignosulfonate is always higher than that of sodium dodecyl sulfate. When the concentration of coal water slurry is $67 \%$, the viscosity of the sodium dodecyl sulfate CWS is 986 $\mathrm{mpa} \cdot \mathrm{s}$, and the viscosity of the sodium lignosulfonate CWS is $1001 \mathrm{mpa} \cdot \mathrm{s}$. According to the flow state, the flow of coal water slurry is divided into four grades of A, B, C and D. The classification standards are as follows: A-uninterrupted flow; B-discontinuous flow, thick flow; $\mathrm{C}$ - discontinuous flow, a thin paste; D - non flow ${ }^{[11]}$. The flow state of sodium lignosulfonate CWS is A, and the flow state of sodium dodecyl sulfate CWS is B. Sodium lignosulfonate can better reduce the wettability of coal surface, more easily adsorbed on the surface of coal particles, and improve the hydrophilicity of the coal water interface.

Adding $0.4 \%, 0.6 \%, 0.8 \%, 1.0 \%$ and $1.2 \%$ different content additives to coal water slurry with a concentration of $67 \%$. The apparent viscosity was measured, as shown in Figure 3.

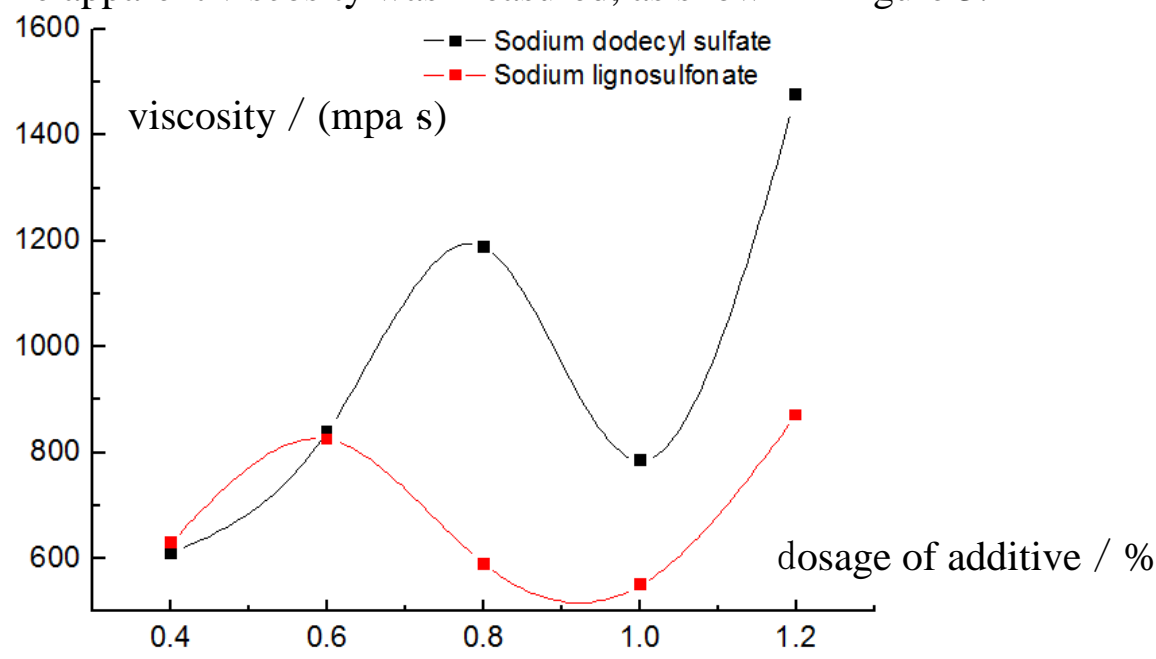

Figure 3 The effect of different additives on the apparent viscosity of coal water slurry

From Figure 3, it can be concluded that the viscosity of CWS increased in the $0.4 \% \sim 0.6 \%$ stage under the $67 \%$ of CWS concentration. The viscosity of coal water slurry prepared by sodium lignosulfonate decreased from $0.6 \%$ to $1 \%$, and reached the lowest value at $1 \%$. The viscosity of coal water slurry prepared by sodium dodecyl sulfate increased first from $0.6 \%$ to $0.8 \%$ and then decreased from $0.8 \%$ to $1 \%$. The viscosity of two kinds of coal water slurry increased when the dosage of the additive is in the $1 \% \sim 1.2 \%$ stage, and the viscosity reached the highest at $1.2 \%$. When the dosage of the additive is not sufficient to make the hydrophobic surface of the pulping coal into a hydrophilic surface, the surface of the coal particles also maintains some original hydrophobic area. Because of the relatively low density of particles on the surface of hydrophilic groups, so no matter from the formation of electrostatic double layer after repulsion, or the formation of water film thickness, and the dispersant adsorbed on the coal surface generated by the steric effect, which is conducive to the particle dispersion factors are difficult to achieve the best. On the contrary, aggregation caused by the hydrophobic interaction between coal particles and Fan Dehua gravity still has certain advantages, under this condition, not only the amount of free water contained in the aggregate structure, but also due to the increased contact chance between coal particles, inevitably lead to increased movement resistance, this is the main reason for the high viscosity of the slurry when the dosage of the additive is low ${ }^{[12]}$.

Stability analysis of coal water slurry

The of coal water slurry can be judged by the water separation rate after a period of time. Selection of coal water slurry with an additive amount of $0.6 \%$. Coal water slurry is placed in $12 \mathrm{~h}$, $24 \mathrm{~h}, 36 \mathrm{~h}, 48 \mathrm{~h}, 60 \mathrm{~h}$ and $72 \mathrm{~h}$ respectively. The amount of free water precipitation is shown in Figure 
4.

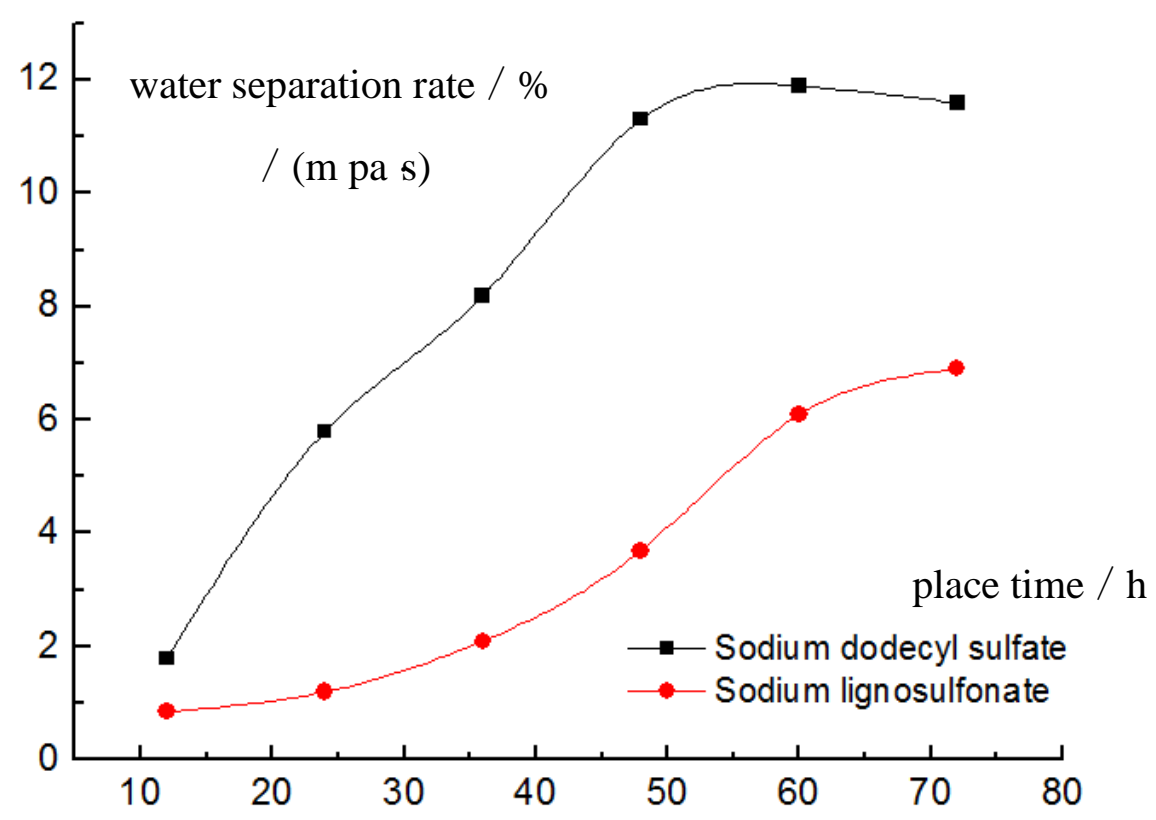

Figure 4 Study on the water separation rate of coal water slurry after a period of time

From Fig. 4, we can see that the water separation rate of coal water slurry with sodium dodecyl sulfate is always higher than that of coal water slurry with sodium lignosulfonate. Before 60h, the water separation rate of two kinds of coal water slurry has been increased, and the difference of water separation rate is the biggest at $48 \mathrm{~h}$. The electrostatic repulsion of coal water slurry added with sodium lignosulfonate is obviously stronger than that of coal water slurry added sodium dodecyl sulfate. The CWS with sodium lignosulfonate better organize the aggregation of coal particles to make it better dispersed. It is concluded that the hydrophilicity of sodium lignosulfonate is better and the stability is higher ${ }^{[13]}$.

\section{Conclusions}

The electrostatic repulsion of coal water slurry added with sodium lignosulfonate is stronger than that of coal water slurry added sodium dodecyl sulfate, which makes it better dispersed. Sodium lignosulfonate, as a polypolymer compound, has strong spatial network structure. Sodium lignosulfonate has greater steric hindrance on coal surface, better hydrophilicity and higher stability.

\section{Acknowledgements}

Fund Project: Gansu provincial key discipline construction projects of Applied Chemistry (No. GSKS201304 AC).

\section{References}

[1] Jia Ming Sheng, Chen En Jian. Characteristics and application fields of coal water slurry instead of oil combustion technology [J]. Industrial heating, 2003, 32(1):8-11.

[2] Du Xiao Ru, Li Guang Mei, Huang Xin, etc. The technology of coal water slurry and the way to improve the pulp property of the hard pulping coal [J]. Coal technology, 2010, 29(1):176-178.

[3] Cen Ke Fa, Yao Qiang, Cao Xin, etc. Theory and application technology of coal water slurry combustion flow, heat transfer and gasification [M]. Zhe Jiang University Press, 1997.

[4] Zhang Yan Lin, Qiu Xue Qing, Wang Wei Xing. Development trend of coal water slurry additive [J]. Modern chemical industry, 2004, 24(3):16-19. 
[5] Guo Zhao Bing, Wu Guo Guang. Research status and development trend of coal water slurry additive [J]. Clean coal technology, 2001, 7(1):32-34.

[6] Ran Ning Qing, Yu Ding Ji. Dispersive effect of sodium methylene naphthalene sulfonic acid sodium p-styrenesulfonate - sodium maleate on coal water slurry [J]. Journal of Nanjing University (natural science), 1999(5):643-647.

[7] Wang Qun Ying, Li Han $\mathrm{Xu}$. The structure of coal water slurry dispersant and its influence on the surface properties of coal [J]. Journal of Anhui University of Science and Technology (natural science), 2002, 22(3):37-39.

[8] Li Yan Chang, Zhou Zhi Qiang, Cheng Jun, etc. Influence of physicochemical properties of coal on its pulp performance [J]. Coal conversion, 2009, 32(3): 35-39.

[9] Shou Chong Qi, Zhao Chun Bin, Jia Hai Bo, etc. Study on the additive and its stability of coal water slurry [J]. Chemical industry of daily use, 2003, 33(2):120-123.

[10] Song Jun Wang, Guo Rui, Lu Kai, etc. Study on the modification and application of sodium lignosulfonate [J]. Coal conversion, 2012, 35(4): 56-59.

[11] Sun Mei Jie, Tu Ya Nan, Chu Tian Cheng, etc. Study on the effect of Sodium Lignosulfonate on the stability of coal water slurry [J]. Coal engineering, 2014, 46(11): 109-112.

[12] Zou Li Zhuang, Zhu Shu Quan, Zhi Xian Hua, etc. Study on the interaction between different coal water slurry additives and coal - Influence of the dosage of dispersant on the rheological properties of coal water slurry (IV) [J]. Journal of China University of Mining and Technology, 2004, 33(4):370-374.

[13] Deng Shao Bo, Cao Qing, Jin Li E, etc. Study on the modification of sodium lignosulfonate and the dispersion of coal slurry [J]. Coal conversion, 2014, 37(2): 33-36. 